\title{
A Viable Passive Optical Network Design for Ultrahigh Definition TV Distribution
}

\author{
Shahab Ahmad Niazi, Xiaoguang Zhang, Lixia Xi, Abid Munir, \\ Muhammad Idrees, and Yousaf Khan
}

Institute of Information Photonics and Optical Communications, State Key Laboratory of Information Photonics and Optical Communication, Beijing University of Posts and Telecommunications, 10 Xitucheng Road, Beijing 100876, China

Correspondence should be addressed to Shahab Ahmad Niazi; shahabniazi@yahoo.com

Received 30 January 2013; Revised 15 April 2013; Accepted 22 April 2013

Academic Editor: Jung Huang

Copyright (C) 2013 Shahab Ahmad Niazi et al. This is an open access article distributed under the Creative Commons Attribution License, which permits unrestricted use, distribution, and reproduction in any medium, provided the original work is properly cited.

\begin{abstract}
International Telecommunication Union (ITU) has recently standardized ultrahigh definition television (UHD-TV) with a resolution 16 times more than the current high definition TV. An increase in the efficiency of video source coding or in the capacity of transmission channels will be needed to deliver such programs by passive optical network (PON). In this paper, a high capacity integrated PON infrastructure is proposed to overlay ultrahigh definition television by a complete passive coexistence of 10G-PON (XG-PON) and single carrier directly modulated, duo-binary 40G-PON (XLG-PON) signal. The simulation results show error-free transmission performance and further distribution to 32 optical network units (ONUs) on broadcast basis with negligible power penalty over $20 \mathrm{~km}$ of bidirectional standard single mode fiber.
\end{abstract}

\section{Introduction}

Service providers and telecom operators are very keen to offer triple-play (video, voice, and data) service on single common broadband passive optical network (PON) infrastructure. The present cost model for optical access only reflects the broadband connections to end users but it is obvious that this fiber setup should be used to distribute the other services such as wireless access and HDTV signals. It is very important to share the high installation cost of fibers between diversified revenue streams. Therefore, the engineering and legal liability to install the fiber network must be supported by as many services as possible $[1,2]$. Internet protocol television (IPTV) is becoming a common service for many IP service providers, especially for live television and for video on demand (VOD) [3]. IPTV not only provides the access to the channel programs as does the traditional TV, it also provides interactivity with the network. Moreover, it provides end-toend service quality compared to internet video that works on "best effort" fashion with no end-to-end service management and quality of service considerations. However, the next challenge for IPTV providers is to enhance the bandwidth capacity to deliver contents in stereoscopic video, which has gained popularity for 3D vision films [4]. At present, the major concern with the IPTV service is the delay in channel selection for live broadcast TV. The channel change response time (CCRT) is defined as the time delay between a viewer asking for a channel change by pressing some buttons on a remote control unit and the display of the selected channel on the TV screen [5]. The CCRT is affected by network operations such as admission control, multicast distribution at routers, and video processing (decoding and buffering) at network elements. Network delay is the time interval that the first video frame of a requested channel arrived after the internet group management protocol (IGMP) leave/join process completed $[6,7]$. Therefore, the multicast approach is causing a delay delivery of live IPTV contents. The other considerable delay is caused by computational complexity and quantization effects of decoding process at the set-top box. The H.264/MPEG-4 encoding process is employed to reduce the size of TV channel to make it adaptable for low bandwidth systems. Normally, a high definition television application compressed at a constant bit rate of $12 \mathrm{Mbit} / \mathrm{s}$ [8]. The settop box (STB) is a device that connects to a television and 


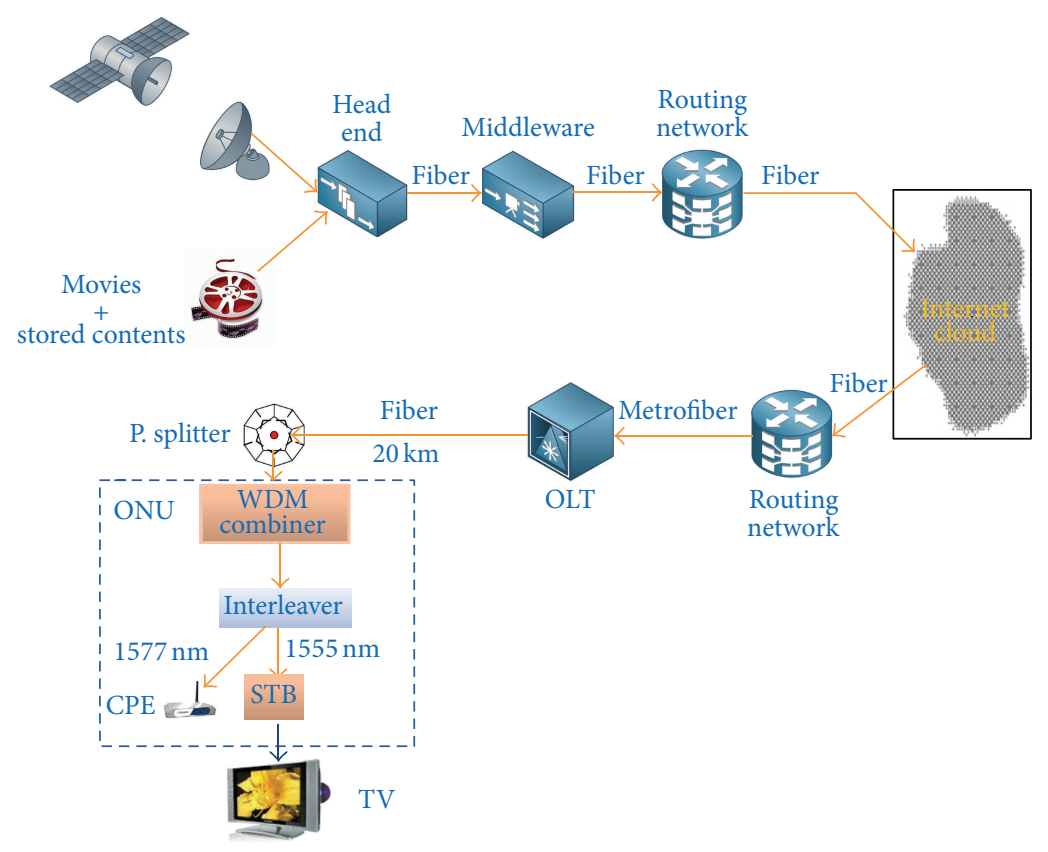

FIGURE 1: The principle diagram of typical setup of IPTV distribution.

an external source of signal, performs encoding/decoding, and provides the content which is then displayed on the television screen. It also works as a minirouter and completes the interactive functions directly through the IP packets interactive mode (HTTP, RTSP, IGMP).

The 8K UHDTV systems are expected to become available till 2017-2020 in South Korea and Japan [9]. The substantial improvement in the efficiency of video source coding or quite noticeable increase in the capacity of transmission channels will be required to deliver such programs by PON [10]. A highly complex source encoder will be required to reduce the $3 \mathrm{Gbit} / \mathrm{s}$ data rate of UHD-TV into $10-20 \mathrm{Mbit} / \mathrm{s}$ range. It may require either quite complicated processing at the set-top box or compromise on some quality features of UHD-TV. The complex processing at the set-top box will result in adding delay in channel selection. Therefore, a large bandwidth system is required to simplify the work of source encoding so that $50-100 \mathrm{MB} /$ channel will easily be supported by future PON networks. Furthermore, the increase in bandwidth will also allow broadcasting of all live TV channels at the same time to reduce the channel latency problem of present multicast propagation. IPTV over GPON and GEPON is proposed $[11,12]$ but it is based on multicasting and legacy TV transmission.

On the other hand, the coexistence of 10G-PON and single wavelength $40 \mathrm{G}-\mathrm{PON}$ is not previously proposed. A prototype network is introduced for TWDM PON on Huawei based system with intensity modulator and offline processing $[13,14]$, where a coexistence of $10 \mathrm{G}$ and $40 \mathrm{G}$ PON systems are indicated, but this $40 \mathrm{G}-\mathrm{PON}$ is comprised of four wavelengths of $10 \mathrm{G}$ data rate as per the proposed standardization of NG-PON2. Moreover, this system is just presenting the combined propagation of XG-PON and XLG$\mathrm{PON}$, whereas their generation is by two complete different modules. In [15], some features of XG-PON1 (10G-PON) and NG-PON2 (40G-PON) are compared but the options for implementations of NG-PON2 or coexistence generation and transmission were not discussed.

In this paper, to the best of our knowledge, a novel coexistent 10G-PON and single wavelength 40G-PON with entirely passive and optical plant are demonstrated to achieve high bandwidth for overlay of UHD-IPTV. After this brief introduction, Section 2 describes the simulation setup and working principle of the architecture of our proposed scheme, Section 3 presents simulation results and analysis of these results and finally Section 4 summarizes the paper.

\section{Simulation Setup and Working Principle}

A typical optical transport network layout for the delivery of IPTV services is presented in Figure 1. Live television content from sources such as satellite, analog off the air, or direct feeds from broadcasters are aggregated at the head end. The middleware provides management functions, channel mapping, electronic program data, content (both music and video) on demand (COD), online games, and internet connectivity $[16,17]$. This content is then distributed over the core transport network to the central offices (CO) located in metropolitans/cities. The CO further connected to optical line terminals (OLTs) through metro area fibers. To achieve very high bandwidth for delivery of UHD-IPTV, a proposed schematic architecture of coexistent XG-PON and single carrier XLG-PON is shown in Figure 2. In this scheme, an optical line terminal (OLT) connected to the remote node (RN) by $20 \mathrm{~km}$ of standard single mood fiber to deliver multimedia services to 32 ONUs. The OLT consists of an optical duobinary XLG-PON downstream transmitter 


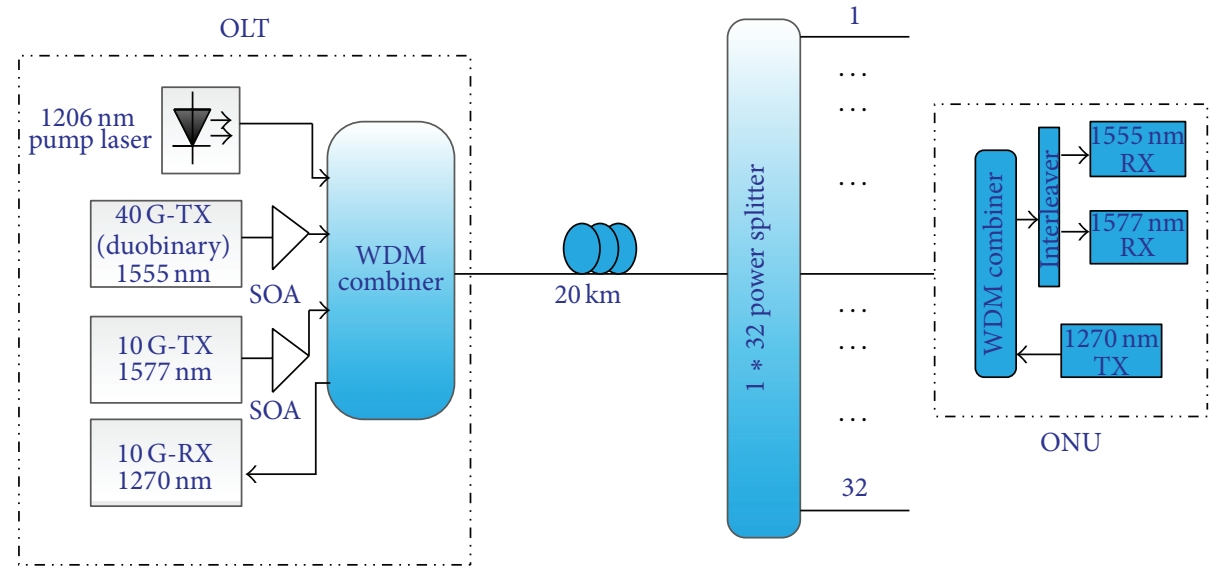

FIGURE 2: The schematic diagram used for coexistence generation and transmission of XG-PON and XLG-PON.

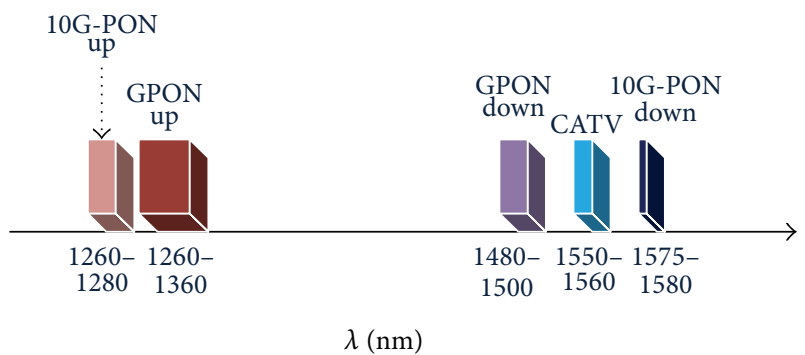

Figure 3: ITU bandwidth specifications for GPON and XG-PON.

at $1555 \mathrm{~nm}$, a distributed feedback laser (DFB) at $1577 \mathrm{~nm}$ as XG-PON downstream transmitter, and avalanche photo diode (APD) as XG-PON upstream receiver. A pump laser is also added at OLT to boost XG-PON upstream signal to avoid the insertion of amplifier at subscriber end. The pump laser is coupled into the feeder fiber to provide the distributed Raman gain for upstream XG-PON signal band (1260$1280 \mathrm{~nm}$ ) at wavelength $1206 \mathrm{~nm}$. The choice of wavelengths is made to ensure compatibility with the wavelength band specifications for XG-PON and GPON signals as defined in ITU-T standards [18, 19], also depicted in Figure 3.

For high-speed optical $40 \mathrm{Gbit} / \mathrm{s}$ XLG-PON link, chromatic dispersion (CD) limits the transmission distance to a few kilometers without dispersion compensation [20]. In this setup, a three-level intensity duobinary modulation is applied to reduce the impacts of chromatic dispersion for XLG-PON to avoid the need for dispersion compensation modules in access network. A duobinary coding process is illustrated in Figure 4 . The power spectral density of duobinary signals is more concentrated around the optical carrier resulting in reduced bandwidth requirements for the system that helps to increase $\mathrm{CD}$ tolerance about three times more than nonreturn to zero (NRZ) [21]. The duobinary data can be generated at the transmitter by sending NRZ data through a "delay and add" filter, which has a $z$-transform of $1+z^{-1}$ and

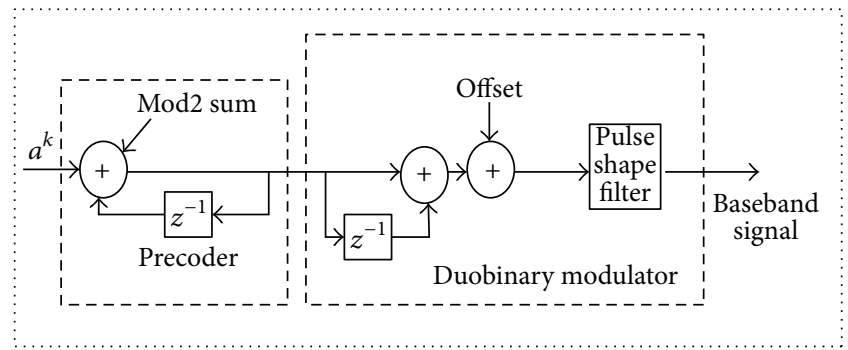

FIGURE 4: Duobinary modulation encoding schematic diagram.

can be approximated by a low-pass filter [22]. The precoder involves a delay of $2 T_{b}$ seconds:

$$
c_{k}=a_{k}-a_{k-2} .
$$

We get an overall frequency response of

$$
H(f)=\left\{\begin{array}{c}
\left.2 j \sin \left(2 \pi f T_{b}\right) \exp \left(-j 2 \pi f T_{b}\right) \longrightarrow|f| \leq \frac{1}{2 T_{b}}\right\} . \\
0 \longrightarrow \text { eleswhere }
\end{array}\right.
$$

The modified duobinary coder consists of the impulse response of two sinc pulses that are time-displaced by $2 T_{b}$ seconds with each other:

$$
h(t)=\frac{2 T_{b}^{2} \sin \left(\pi\left(t / T_{b}\right)\right)}{\pi t\left(2 T_{b}-t\right)} .
$$

To eliminate the possibility of error propagation, data can be precoded propositionally:

$$
d_{k}=b_{k} \oplus d_{k-2} .
$$

A $10 \mathrm{GHz}$ low-pass filter is used to generate $40 \mathrm{Gbps}$ duobinary signal in this simulation setup. The distributed feedback laser diodes (DFB LDs) is modulated at $10 \mathrm{Gbit} / \mathrm{s}\left(2^{31-1}\right)$ 


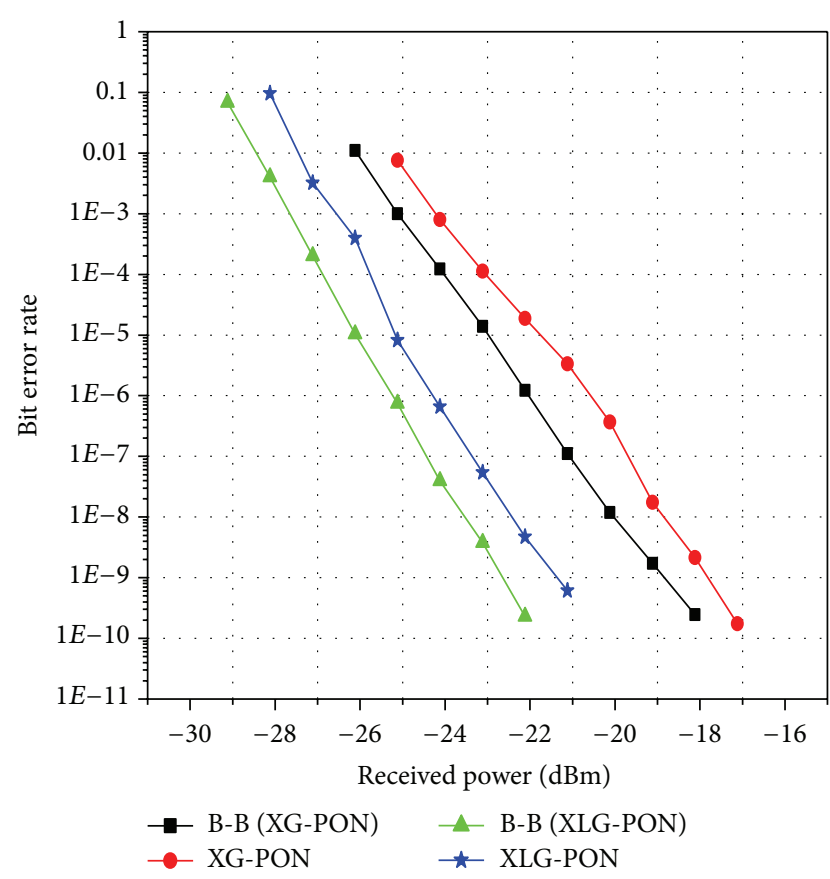

FIGURE 5: Downstream bit error rate as a function of received power back to back signal and after $20 \mathrm{~km}$ propagation of XG-PON and XLG-PON.

pseudorandom binary sequence (PRBS) using pattern generator (PG) to operate at $1577 \mathrm{~nm}$ used as the XG-PON downstream transmitter. The chirp $\Delta v(t)$ of a directly modulated laser (DML) is related to the laser output optical power $P(t)$ through the expression [23]

$$
\Delta v(t)=\frac{\alpha}{4 \pi}\left(\frac{d}{d t}[\ln (P(t))]+\kappa P(t)\right),
$$

where $\alpha$ is the line-width improvement factor and $\kappa$ is the adiabatic chirp coefficient. The output power $P(t)$ is interrelated to the photon density $S(t)$ through the relation

$$
P(t)=\frac{V \eta h v}{2 \Gamma \tau_{P}} S(t) .
$$

The photon density $S(t)$ is calculated by the small signal, single mode laser rate equations in this simple form

$$
\begin{gathered}
\frac{d S(t)}{d t}=\frac{\Gamma g_{0}\left(N(t)-N_{0}\right)}{1+\varepsilon S(t)} S(t)-\frac{S(t)}{\tau_{P}}+\frac{\Gamma \beta N(t)}{\tau_{C}}, \\
\frac{d N(t)}{d t}=\frac{I(t)}{e V}-\frac{N(t)}{\tau_{c}}-\frac{g_{0}\left(N(t)-N_{0}\right)}{1+\varepsilon S(t)} S(t), \\
\frac{d \phi}{d t}=\frac{\alpha}{2}\left[\Gamma g_{0}\left(N(t)-N_{0}\right)-\frac{1}{\tau_{P}}\right],
\end{gathered}
$$

where $I(t)$ is the current waveform injected in the active layer, $N(t)$ is the carrier density, $v$ is the optical frequency, $h$ is the Plank's constant, $\eta$ is the differential quantum efficiency, $\Gamma$ is the confinement factor, $N_{0}$ is the carrier density at

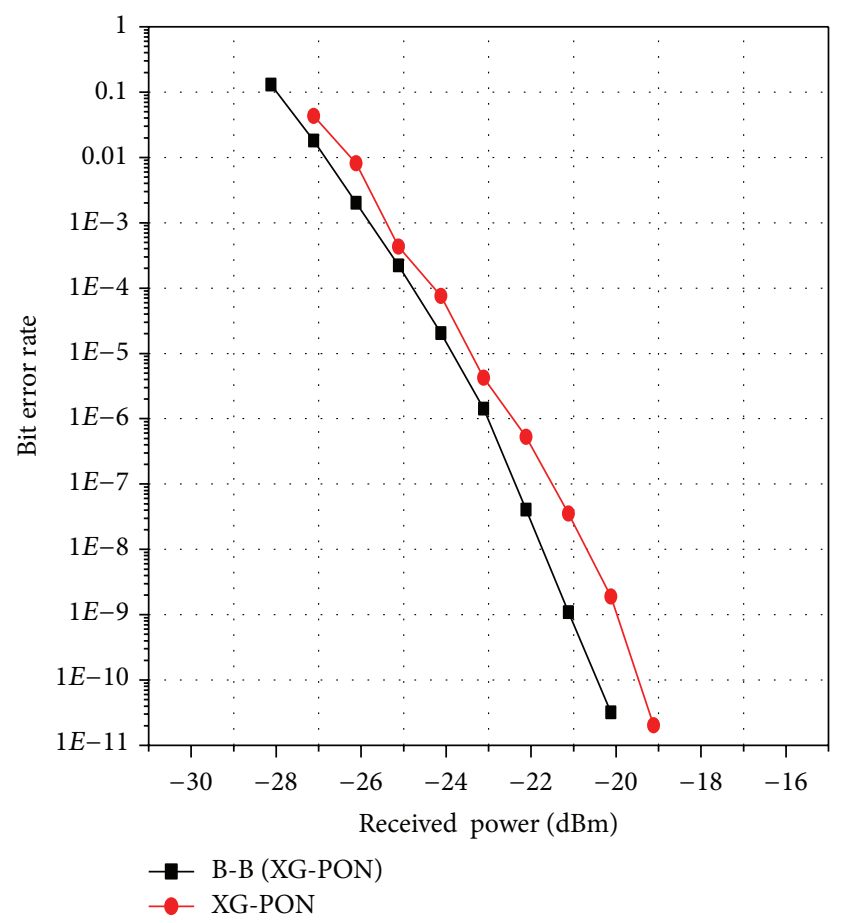

FIGURE 6: Upstream bit error rate as a function of received power for back to back and $20 \mathrm{~km}$ propagation of XG-PON.

transparency, $\beta$ is the fraction of spontaneous emission noise coupled into the lasing mode, $g_{0}$ is the differential gain coefficient, $\varepsilon$ is the nonlinear gain compression factor, $\tau_{p}$ is the photon lifetime, $\tau_{c}$ is the carrier lifetime, and $V$ is the volume of the active layer. One has

$$
\kappa=\frac{2 \Gamma}{\eta h v V} \varepsilon .
$$

Hence, to compensate the losses at high data rates, two low-cost semiconductor optical amplifiers (SOAs) for XGPON and XLG-PON are integrated with downstream (DS) transmitters at the OLT into the feeder fiber to boost down signal powers, respectively. A WDM combiner is employed to combine the XG-PON and XLG-PON downstream signals along with Raman pump laser. After $20 \mathrm{~km}$ propagation, a $1 \simeq 32$ power splitter is used to serve 32 ONUs on power sharing basis in downstream. A built-in fiber Bragg grating (FBG) in WDM combiner is able to filter out undesired Raman ASE noise outside of the US signal bands to improve the transmission performance and mitigate the Raleigh back scattering impacts $[24,25]$. At optical network unit (ONU), an optical interleaver is used to separate the optical power of UHD-TV overlaid XLG-PON from XG-PON signal. Two Bessel filters of order one and bandwidth of $10 \mathrm{GHz}$ at central wavelengths of $1555 \mathrm{~nm}$ and $1577 \mathrm{~nm}$, respectively, are applied to reduce the noise before bit error rate estimation. After photo detection, XLG-PON signal is brought to the television set using a set-top box (STB) that decrypts the signal and assembles the packet information into a viewable program to further convert IP packets into TV contents. 


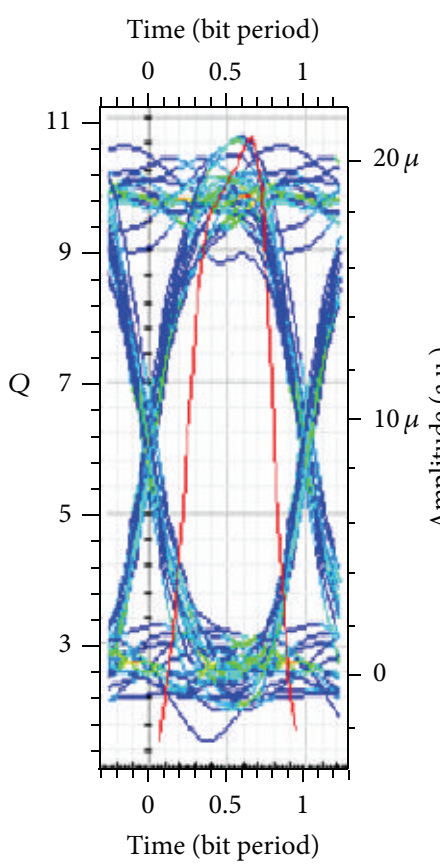

(a)

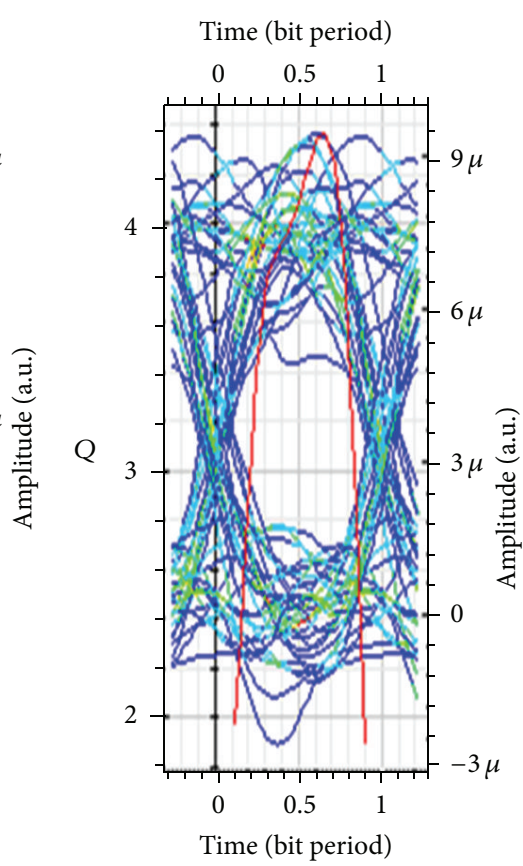

(b)

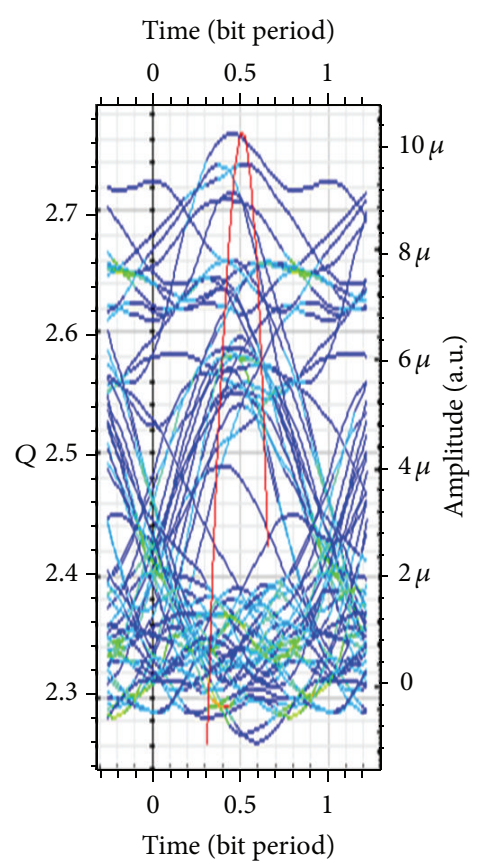

(c)

FIGURE 7: Simulated eye diagrams of the data demodulated for (a) DS XG-PON, (b) US XG-PON, and (c) XLG-PON signals after 20 km transmission.

In uplink, an electroabsorption modulated laser at $1270 \mathrm{~nm}$ is used as XG-PON upstream transmitter. The upstream EML is modulated at $10 \mathrm{Gbit} / \mathrm{s}\left(2^{31-1}\right)$ PRBS using a PG with output power $0 \mathrm{dBm}$ at $1270 \mathrm{~nm}$. A $10 \mathrm{G}$ APD with a band-pass filter is used as XG-PON upstream receiver at OLT to receive US signal. Since only passive splitter is used at RN, the outside fiber plant is entirely passive.

\section{Simulation Results}

The simulation work is performed to testify the coexistence generation and transmission of XG-PON and XLG-PON signals. The power for $1206 \mathrm{~nm}$ pump laser was fixed at $500 \mathrm{~mW}$ that was optimized to ensure 10G-PON error-free upstream operation. The bit error rate (BER) as a function of received optical power for downstream transmission for XG-PON and XLG-PON signals back to back and $20 \mathrm{~km}$ reach with total 1:32 split is shown in Figure 5. For $1577 \mathrm{~nm}$ downstream, there was about $1 \mathrm{~dB}$ power penalty after $20 \mathrm{~km}$ transmission with total link loss of $34 \mathrm{dBm}$, while XLGPON has shown the power penalty of $1.3 \mathrm{~dB}$ with link loss remaining at $37 \mathrm{dBm}$. In upstream, a power penalty of $1 \mathrm{~dB}$ for XG-PON relative to back to back experienced at BER of $1 E-9$ after propagating the distance of $20 \mathrm{~km}$ is shown in Figure 6, whereas clear eye diagrams after downstream XG-PON and XLG-PON and upstream XG-PON after $20 \mathrm{~km}$ traversal are shown in Figure 7. Therefore, it is evident from the above results that an error free transmission has been achieved for both downstream and upstream direction. In this scheme, $1555 \mathrm{~nm} 40 \mathrm{G}$ signal is propagated only in downstream with enough bandwidth to support live broadcast of many hundreds UHD-IPTV channels without involving very reduced compression ratios. The IPTV services like VOD, community TV, time shift television, parent control, electronic program guide that requires backward communication with the network operator and service provider for service selection, termination, and payment information can be accommodated by reserving some bandwidth on upstream 10G-PON. However, this backward communication ability can be easily provided by reserving some bandwidth on upstream 10GPON. If separate wavelength is dedicated for backward communication, it will not only be a wastage of network resources but also incur a huge extra cost.

\section{Conclusion}

This coexistence scheme of XLG-PON and XG-PON can be used for the overlay of UHD-IPTV for simultaneous transmission of hundreds of channels at almost unnoticeable delay and quite less processing involved in set-top box. Therefore, it is a bright opportunity to provide the live UHDTV service with no latency and with all the existing and future auxiliary services of IPTV with minimum cost involved in ONU.

\section{Acknowledgment}

The work is partially supported by research fund for the doctoral program of higher education (Grant no. 20110005110014). 


\section{References}

[1] IPTV Service Architecture, ITU-T FG IPTV-ID-0094, July 2006.

[2] A. Y. Lee, A. Modarress, and S. Mohan, "Convergence of application services in next-generation networks," IEEE Communications Magazine, vol. 50, no. 3, pp. 16-17, 2012.

[3] C. Meusburger, D. A. Schupke, and A. Lord, "Optimizing the migration of channels with higher bitrates," Journal of Lightwave Technology, vol. 28, no. 4, pp. 608-615, 2010.

[4] H. Kalva, L. Christodoulou, L. Mayron, O. Marques, and B. Furht, "Three dimensional television services," in Encyclopedia of Multimedia, part 20, pp. 864-868, Springer, New York, NY, USA, 2008.

[5] J. Yoon, S. Park, and S. Choe, "Implementation of EIGMP for fast IPTV channel change in GEPON," IEEE Transactions on Consumer Electronics, vol. 57, no. 2, pp. 484-491, 2011.

[6] E. Lee and S. Park, "Internet group management protocol for IPTV services in passive optical network," IEICE Transactions on Communications, vol. E93-B, no. 2, pp. 293-296, 2010.

[7] U. Oh, S. Lim, and H. Bahn, "Channel reordering and prefetching schemes for efficient iptv channel navigation," IEEE Transactions on Consumer Electronics, vol. 56, no. 2, pp. 483-487, 2010.

[8] O. Issa, F. Speranza, W. Li, and H. Liu, "Estimation of time varying QoE for high definition IPTV distribution," in Proceedings of the IEEE Consumer Communications and Networking Conference (CCNC '12), pp. 326-330, 2012.

[9] E. Puigrefagut, "HDTV and beyond," in Proceedings of the ITU Regional Seminar on Transition to Digital Terrestrial Television Broadcasting and Digital Dividend, Budapest, Hungary, November 2012.

[10] "Parameter values for ultra-high definition television systems for production and international programme exchange," Recommendation ITU-R BT-2020, 2012.

[11] H. Ikeda, J. Sugawa, Y. Ashi, and K. Sakamoto, "High-definition IPTV broadcasting architecture over gigabit-capable passive optical network," in Proceedings of the 50th Annual IEEE Global Telecommunications Conference (GLOBECOM '07), pp. 22422246, November 2007.

[12] J. Wei, "The VoD services carried by hybrid PON+EoC networking," in Proceedings of the 2nd IEEE International Conference on Broadband Network and Multimedia Technology (ICBNMT '09), pp. 467-471, October 2009.

[13] Y. R. Ma, Y. B. Qian, G. K. Peng et al., "Demonstration of a $40 \mathrm{~Gb} / \mathrm{s}$ time and wavelength division multiplexed passive optical network prototype system," in Proceedings of the Optical Fiber Communication Conference and Exposition and the National Fiber Optic Engineers Conference (OFC/NFOEC '12), 2012.

[14] Y. Q. Luo, X. J. Yan, and F. Effenberger, "Next generation passive optical network offering $40 \mathrm{~Gb} / \mathrm{s}$ or more bandwidth," in Proceedings of the Asia Communications and Photonics Conference (ACP '12), OSA, 2012.

[15] F. Effenberger, "XG-PON1 versus NG-PON2: which one will win," in Proceedings of the European Conference and Exhibition on Optical Communication (ECOC '12), OSA, 2012.

[16] M. El-Sayed, Y. Hu, S. Kulkarni, and N. Wilson, "Access transport network for IPTV video distribution," in Proceedings of the Optical Fiber Communication Conference, and the National Fiber Optic Engineers Conference (OSA/NFOEC '06), OSA, 2006.
[17] S. Song, H. Moustafa, and H. Afifi, "Advanced IPTV services personalization through context-aware content recommendation," IEEE Transactions on Multimedia, vol. 14, no. 6, pp. 15281537, 2012.

[18] ITU-T Series Recommendation G.987.2, 2010.

[19] ITU-T Series Recommendation G.984.5, 2008.

[20] C. W. Chow, L. Xu, C. H. Yeh, H. K. Tsang, W. Hofmann, and M. C. Amann, "40-Gb/s upstream transmitters using directly modulated 1.55- $\mu \mathrm{m}$ VCSEL array for high-split-ratio PONs," IEEE Photonics Technology Letters, vol. 22, no. 5, pp. 347-349, 2010.

[21] D. V. Veen, D. Suvakovic, H. Chow, V. Houtsma, E. Harstead, and P. Vetter, "Options for TDM PON beyond 10G", in Proceedings of the Advanced Photonics Congress, OSA, 2012.

[22] J. H. Sinsky, A. Konczykowska, A. L. Adamiecki, F. Jorge, and M. Duelk, " $39.4 \mathrm{~Gb} / \mathrm{s}$ duobinary transmission over $24.4 \mathrm{~m}$ of coaxial cable using a custom indium phosphide duobinaryto-binary converter integrated circuit," IEEE Transactions on Microwave Theory and Techniques, vol. 56, no. 12, pp. 3162-3169, 2008.

[23] I. Tomkos, I. Roudas, R. Hesse, N. Antoniades, A. Boskovic, and R. Vodhanel, "Extraction of laser rate equations parameters for representative simulations of metropolitan-area transmission systems and networks," Optics Communications, vol. 194, no. 1-3, pp. 109-129, 2001.

[24] S. Stevan, A. Teixeira, G. M. T. Beleffi, P. Andre, and A. Pohl, "Rayleigh backscattering lasing control based on Raman amplification," IET Optoelectronics, vol. 6, no. 2, pp. 88-93, 2012.

[25] J. S. Jeong, H.-S. Kim, B.-S. Choi et al., "Mitigation of Rayleigh crosstalk using noise suppression technique in 10-Gb/s REAMSOA," Optics Express, vol. 20, no. 24, pp. 26373-26378, 2012. 

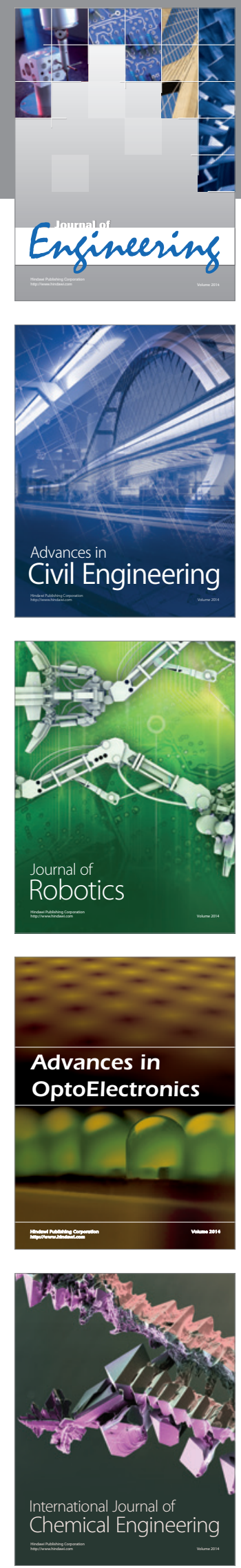

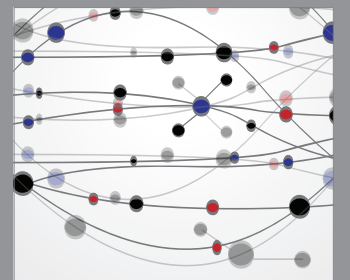

The Scientific World Journal
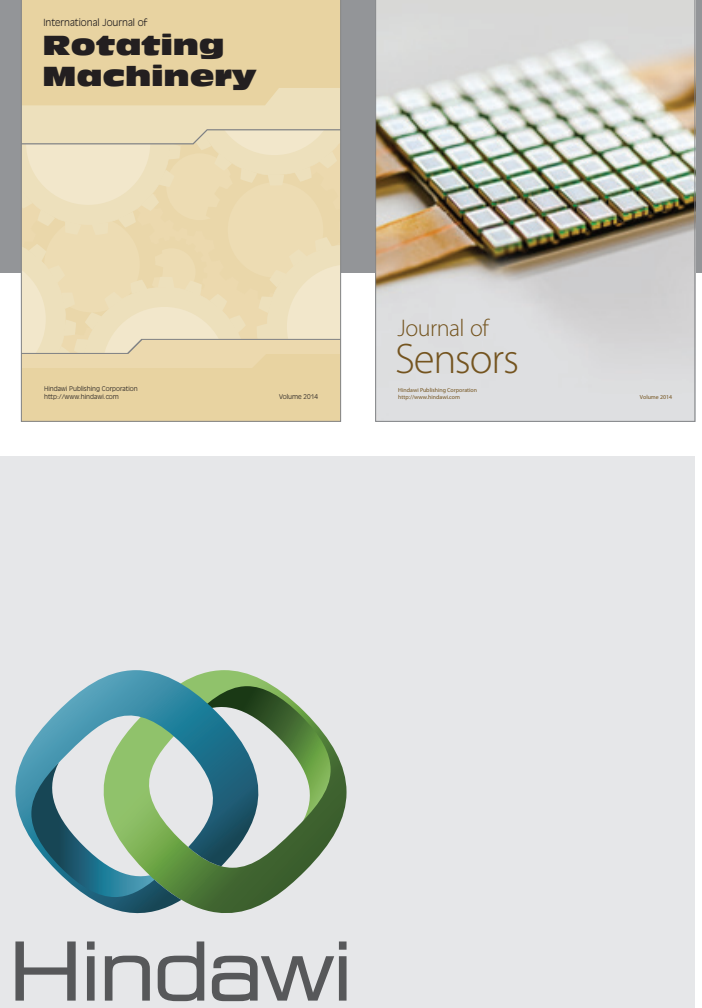

Submit your manuscripts at http://www.hindawi.com
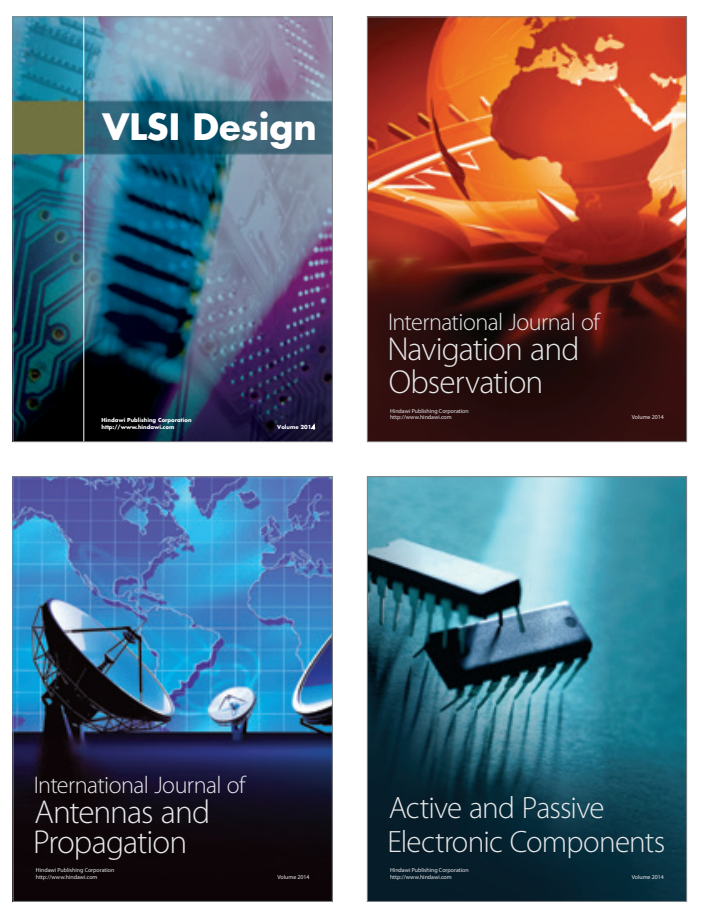
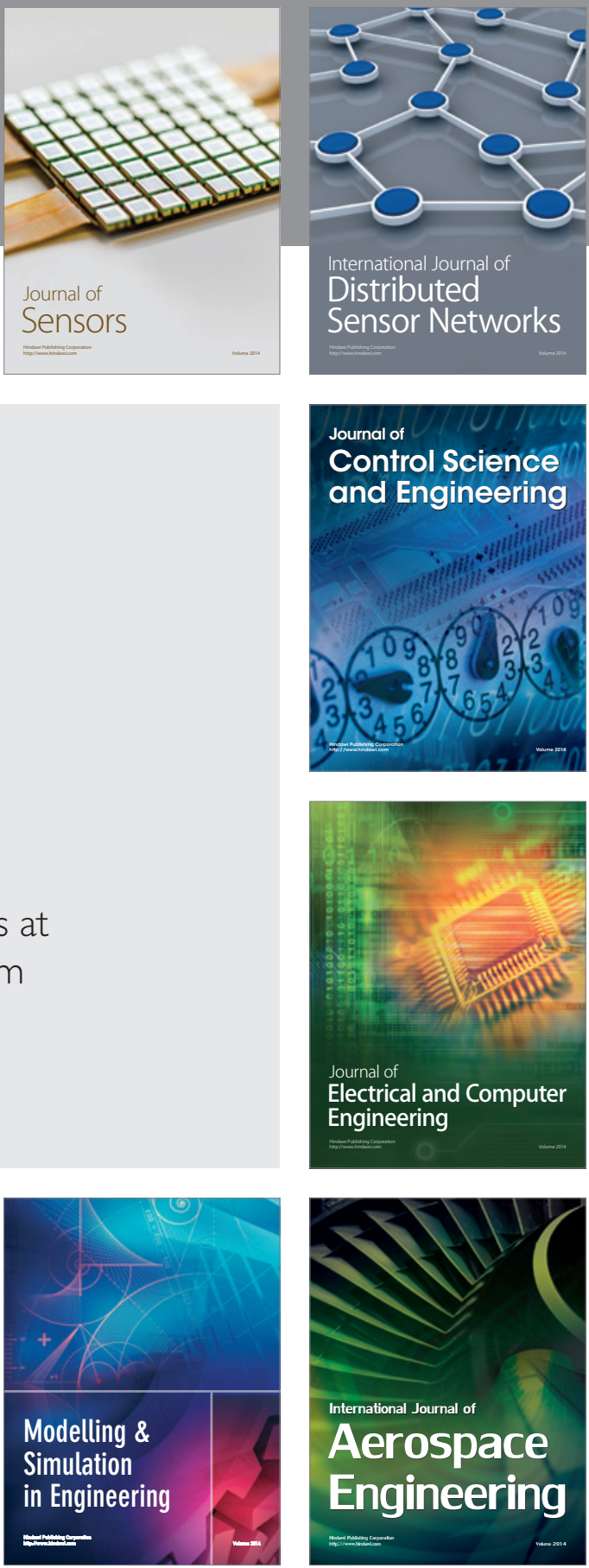

Journal of

Control Science

and Engineering
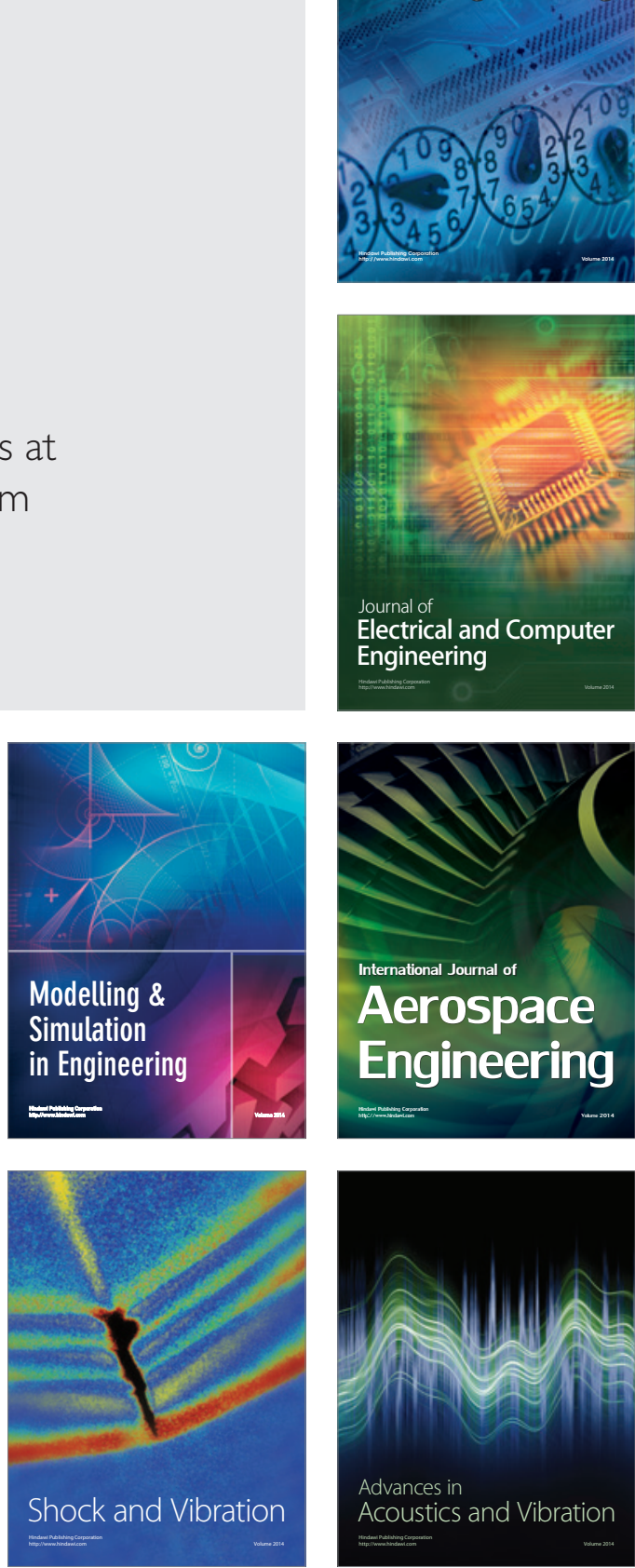